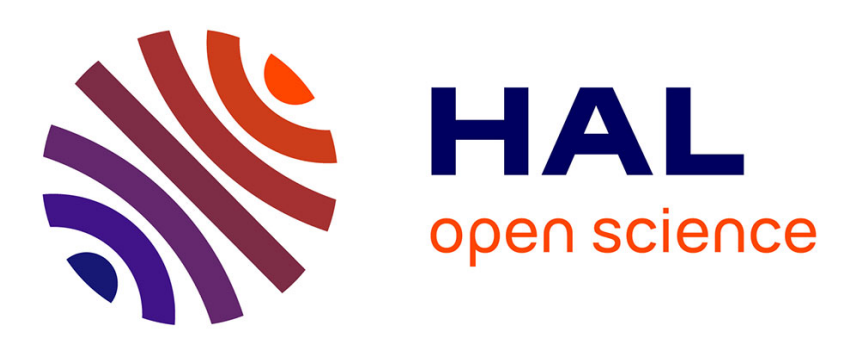

\title{
Diffusion coupling in multiply associating electrolyte solutions
}

\author{
Jean-Pierre Simonin, José Miguel Ramos, José Torres-Arenas
}

\section{To cite this version:}

Jean-Pierre Simonin, José Miguel Ramos, José Torres-Arenas. Diffusion coupling in multiply associating electrolyte solutions. Journal of Molecular Liquids, 2016, 215, pp.69-76. 10.1016/j.molliq.2015.12.020 . hal-01252050

\section{HAL Id: hal-01252050 https://hal.sorbonne-universite.fr/hal-01252050}

Submitted on 7 Jan 2016

HAL is a multi-disciplinary open access archive for the deposit and dissemination of scientific research documents, whether they are published or not. The documents may come from teaching and research institutions in France or abroad, or from public or private research centers.
L'archive ouverte pluridisciplinaire HAL, est destinée au dépôt et à la diffusion de documents scientifiques de niveau recherche, publiés ou non, émanant des établissements d'enseignement et de recherche français ou étrangers, des laboratoires publics ou privés. 


\title{
Diffusion coupling in multiply associating electrolyte solutions
}

\author{
Jean-Pierre Simonin ${ }^{\mathrm{a}, *}$, José Miguel Ramos ${ }^{\mathrm{b}}$, José Torres-Arenas ${ }^{\mathrm{b}}$ \\ ${ }^{a}$ Laboratoire PHENIX, Sorbonne Universités, UPMC Univ Paris 06, UMR 8234, \\ Université P.M. Curie, F-75005, Paris, France \\ ${ }^{b}$ División de Ciencias e Ingenierías, Campus León, Universidad de Guanajuato, León, \\ México
}

- Transient diffusion profiles of zinc(II) ion in the electric field created by major LiCl salt were obtained.

- The process is modeled by accounting for the various effects in play.

- Multiple complexation of zinc by chloride ion, non-ideality and electric field are the dominant effects.

- Deviations from ideality are described within the MSA model.

\begin{abstract}
Diffusion coupling in multiply associating aqueous electrolyte solution is studied. The process is illustrated in the case of zinc(II) ion which forms a set of complexes, $\mathrm{ZnCl}_{n}^{2-n}$ (with $n=1, . ., 4$ ), in the presence of chloride ions. The transport of zinc ion, taken in radioactive form ${ }^{65} \mathrm{Zn}^{2+}$, in the electric field created by a gradient of $\mathrm{LiCl}$, is investigated experimentally by employing an adaptation of the closed capillary technique. The transient diffusion process is modeled by using two different treatments: finite difference (FD) simulation and normal mode (NM) analytic solution. Deviations from ideality are taken into account with the use of the mean spherical approximation (MSA), and the internal electric field is calculated using the dynamical electroneutrality condition. The theoretical FD and NM results are compared with the experimental data about the diffusion of zinc.
\end{abstract}

Keywords: Electrolytes; association; non-ideal; diffusion; mean-spherical approximation (MSA).

\section{Introduction}

Aqueous electrolytes are ubiquitous on earth. They may be found in natural waters (e.g., in oceans, lakes, sediments), in most living beings and in

\footnotetext{
${ }^{*}$ Corresponding author

Email address: jpsimonin@gmail.com. Tel. +33 144273190; Fax +33 144273228. (Jean-Pierre Simonin)
} 
plants, trees,... They have a huge influence on the development and functioning of life on the planet. The motion of ionic species governs the local amount of ions. In this respect, aqueous electrolytes have the particular property that the movements of the ions in a medium are strongly coupled through the effect of electrostatic interactions $[1,2]$.

Diffusion coupling thus can be observed in many natural and industrial domains and systems, such as in geochemistry (when studying diagenetic fluxes in sediments [3, 4]), in biophysics (e.g. for a description of permeation through ionic channels of cells [5]), in engineering processes (e.g., when using membranes for industrial separations [6]), in materials science (e.g., in the study of diffusion of ions in concrete [7] or for the assessment of chemical ageing of concrete and composite materials [8]).

Diffusion coupling in aqueous ionic solutions has been investigated at a fundamental level for a long time [1]. Theoretical descriptions have been developed that assume linear transport theory, in which fluxes are linear functions of forces [9]. Multicomponent diffusion has been examined in the framework of Onsager formalism of irreversible thermodynamics [10].

A phenomenon that has great influence on coupled diffusion in electrolytes is ionic association, consisting either of ion pairing or chemical reaction. In geochemistry, Lasaga first examined the influence of ion pair formation on diagenetic fluxes in marine sediments [4]. Ion pairing is important because it modifies the effective charge of the diffusing species and so changes their response to the internal electric field created by the diffusing ions. A clear example is that of a monovalent tracer ion that associates with a monovalent anion and forms a neutral species that is insensitive to the diffusion field [4].

Some time ago, we investigated the effect of ion pairing caused by magnesium(II) ion on the transient transport of sulfate ion by carrying out experiments in a special diffusion cell (closed capillary) [11]. Later we investigated the effect of a $\mathrm{pH}$ gradient on sulfate and phosphate ions [12]. In these works, the diffusion-reaction equations were solved by using two types of treatments: a 'normal-mode' analysis in which the equations are linearized and solved analytically [13]; and a classical numerical finite-difference method in which time and space are 'sliced' and the equations are solved incrementally in the course of time. In these treatments, the equations were written in the ideal case in which activity coefficients are taken equal to unity. Besides, the associations involved only one type of complex, leading to the ion pair $\mathrm{MgSO}_{4}^{0}$ in the case of $\mathrm{Mg}^{2+}$ and $\mathrm{SO}_{4}^{2-}$ [11], or to the hydrogen sulfate ion $\mathrm{HSO}_{4}^{-}$in the case of sulfate in the $\mathrm{pH}$ gradient. 
In environmental media, ionic pollutants exist in various forms. For instance, toxic heavy metal ions of various valencies such as zinc, cadmium, lead or mercury may form a series of complexes with ions like chloride, nitrate, hydroxide,... The speciation of these ions in the environment is an important issue because complexation modifies the electric charge borne by the ions. This phenomenon modifies the transport of trace metal ions when they are submitted to diffusion electric fields caused by major ions in the environmental medium, and also the physical and chemical behavior of the ion interacting with the environment, e.g. their interactions with colloidal and mineral particles.

Various softwares exist commercially which permit estimations of the speciation for many metal ions in aqueous solutions. One may cite the following which have been developed in various countries: MINTEQ [14], MINEQL+ [15], JCHESS [16], PHREEQC [17] and CHEAQS [18]. The calculation of the speciation is based on a solution to the chemical association equilibria which involve the introduction of thermodynamic association constants (cumulative constants $\beta_{n}$ for $\left.n=1,2,3, \ldots\right)$ and the use of formulas for the computation of the activity coefficients of the species. The softwares rely on previous determinations of the association constants, which can be found in famous books, e.g., in the book by Sillen and Martell [19]) or in the NIST Database [20]. In general, deviations from ideality were computed using equations of the Debye-Hückel type, like the Davies equation. Depending on the values taken for the $\beta_{n}$ 's and on the equation used, softwares may give speciations that differ in magnitude. Because the speciation is generally very difficult to determine experimentally, there is uncertainty in the determination of speciation in electrolyte solutions.

In the present work, we propose to study transient diffusion patterns in the case of a metal cation giving rise to multiple association. The metal cation (in tracer amount) is placed in the electric field created by the diffusion of a major salt. This type of experiment is a variation on the theme of experiments we had carried out with magnesium(II) and sulfate ions [11, 12]. The latter system gave one type of ion pair. In the present work we investigate the case of electrolytes leading to a set of complexes of increasing stoichiometries through a series of stepwise associations.

Moreover, in contrast with our earlier work about coupling diffusion, we now include the effect of deviations from ideality. Accounting for activity coefficients in the treatment has several consequences. It modifies the speciation of the metal cation under study, and therefore the diffusion rate of the 
various forms (free species and complexes). It also introduces a new driving force associated to the variation in space of the activity coefficients of the chemical species, and it modifies the magnitude of the internal diffusion electric field created by the major ions. The experimental observable is the result of these different effects.

In this work, we carried out experiments with the zinc(II) cation. This species has an important role in biological, geochemical and environmental media $[22,23]$. It is well known for giving rise to multiple association with various anions [19] . Here, we used zinc chloride, which can give 4 complexes: $\mathrm{ZnCl}^{+}, \mathrm{ZnCl}_{2}^{0}, \mathrm{ZnCl}_{3}^{-}$and $\mathrm{ZnCl}_{4}^{2-}[19]$. The zinc ion was taken in radioactive form, ${ }^{65} \mathrm{Zn}^{2+}$. A special technique consisting of a silica capillary inserted in a cylinder of scintillating plastic was utilized to observe the transient concentration profiles of the radioactive tracer [24]. The major salt producing the internal electric field was $\mathrm{LiCl}$. It was chosen because it produces a strong electric field, which is due to the greatly differing values of the diffusivities of the two ions $\mathrm{Li}^{+}$and $\mathrm{Cl}^{-}$. The observed experimental transient profiles were modeled by using finite difference (FD) and normal-mode (NM) methods. Deviations from ideality were included in the calculations by using the mean spherical approximation.

The main purpose of this paper is to investigate the validity of the modeling approach for the description of transient diffusion profiles for a multiply associating electrolyte. The theoretical descriptions include diffusion, ionic association and activity coefficients for all species. The maximum concentration of the major salt $(\mathrm{LiCl})$ is sufficiently high to allow an appreciable influence of activity coefficients. However it is limited to $2 \mathrm{M}$ in order to minimize the effect of hydrodynamic interactions and other subtle effects such as reference frame or ionic relaxation [25].

The remainder of this paper is organized as follows. The next section presents the experimental technique employed to probe the diffusion of the tracer in the cell, and the experimental conditions of this study. Then, a theoretical section exposes the basic equations governing the process and the methods used to calculate the observed effect. Next, the experimental data are compared with the results obtained from the model in the Results and Discussion section. Finally a conclusion summarizes the main results of this work and presents some prospects. 


\section{Experimental}

Diffusion experiments on an ion which is a pure $\beta$ emitter can be conducted adequately by using a cell made of perspex and scintillating plastic $[11,12]$. On the other hand if the tracer is both a $\beta$ and $\gamma$ emitter then it is necessary to stop the $\beta$ 's so that only the $\gamma$ emission can be analyzed (otherwise it would be impossible to distinguish the respective effects of the $\beta$ and $\gamma$ radiations in the scintillating plastic). In the present work, the commercially available radioactive isotope of the ion studied here, ${ }^{65} \mathrm{Zn}^{2+}$, is precisely a $\beta$ and $\gamma$ emitter.

In order to solve this difficulty, experiments were carried out by using an adaptation of the closed capillary technique [24]. It is depicted in Figure 1. The cell consists of a silica capillary (sealed at its bottom end) of 3 cm length and ca. $0.8 \mathrm{~mm}$ inner diameter which is introduced into a bore drilled through the center of a cylinder made of scintillating plastic. The plastic cell was designed in such a way that the points $\mathrm{A}, \mathrm{B}$ and $\mathrm{C}$ are on the same straight line [24]. The silica capillary (whose wall is ca. $1 \mathrm{~mm}$ thick) has the property of stopping the $\beta^{+}$particles of $0.33 \mathrm{MeV}$ energy emitted by ${ }^{65} \mathrm{Zn}^{2+}$. In this way, scintillation in the scintillating plastic is produced only by the $\gamma$ radiation. In order to avoid capillarity problems at the mouth of the capillary, the latter was filled on a distance of $2.8 \mathrm{~cm}$ (i.e. $2 \mathrm{~mm}$ below the top of the capillary). Indeed, filling the capillary with liquid up to its mouth was observed to result in a significant loss of liquid in the course of the experiment. The top of the capillary was sealed with a piece of plastic paraffin film (Parafilm) to prevent evaporation. The silica capillary was adjusted within the plastic bore so that the mid-point of the liquid phase coincided with the top of the scintillating plastic cylinder.

The diffusion experiments were conducted as follows. The bottom part of the capillary (up to point $\mathrm{O}$, see Figure 1 ), of length $L$, was filled with a solution containing $1 \mathrm{M}$ or $2 \mathrm{M} \mathrm{LiCl}+10^{-2} \mathrm{M} \mathrm{ZnCl} \mathrm{Zn}_{2}+$ radioactive zinc(II) in tracer amounts (of the order of $10^{-8} \mathrm{M}$ ). Then, the top part of the capillary, of the same length $L$, was filled very carefully with a solution composed of $10^{-2}$ $\mathrm{M} \mathrm{ZnCl}_{2}+$ radioactive zinc(II) at the same concentration. The solutions were introduced in the capillary by using a small plastic Pasteur pipette bearing a thin flexible Teflon tube at its end. This device allowed us to accurately handle very small volumes of liquid, of the order of $10 \mu \mathrm{L}$ (half of the capillary volume). Next, the cell was introduced in a $\beta$ radioactivity counter which determines the number of photons emitted in the scintillating plastic per 


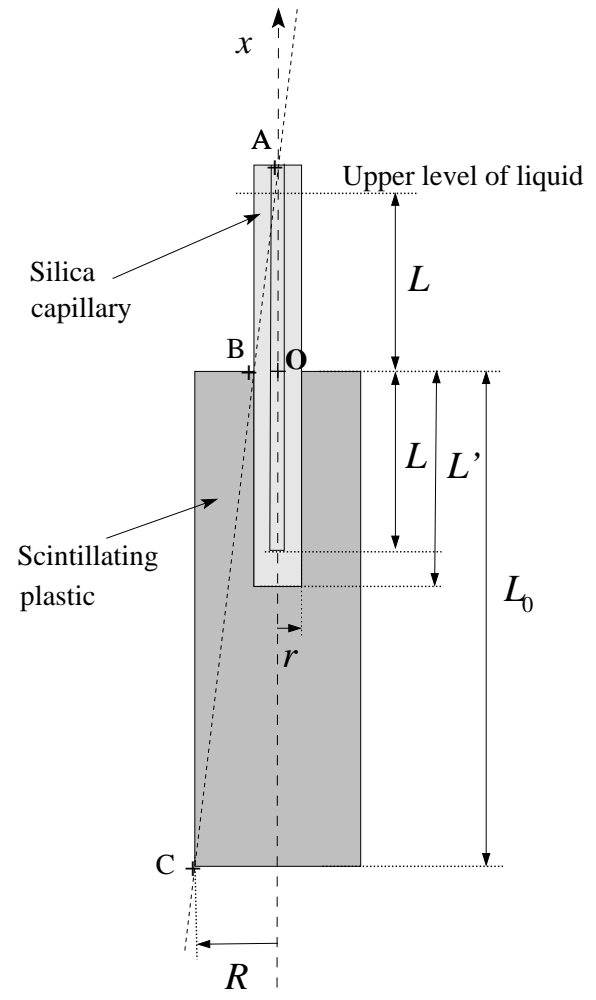

Figure 1: Sketch of the diffusion cell. The points A, B and C are aligned.

time unit. The experiments were conducted with continuous monitoring of radioactivity, at a temperature of $25 \pm 1{ }^{\circ} \mathrm{C}$. They were done in duplicate.

Because it is expected that the $\gamma$ rays can have only one efficient conversion in the plastic, it was shown [24] that the probability of conversion of a $\gamma$ ray is proportional to the length of plastic it crosses. This property allows an explicit calculation of the counting intensity as a function of the tracer concentration profile in the capillary. Then one can measure self-diffusion coefficients of $\gamma$-emitters with this technique (as was done before in the case of a radiolabelled biological molecule [24]), and study diffusion processes like diffusion coupling.

The relative variation of the radioactivity intensity measured by the counter may be written as,

$$
\rho(t) \equiv \frac{I(t)}{I(t=0)}-1
$$


with $I(t)$ the intensity measured by the photomultipliers at time $t$, so that $\rho(t=0)=0$. This intensity is produced by the radioactive tracer in its various forms. At a given position $x$ and time $t$, we denote this total tracer concentration by $C_{T}(x, t)$.

With the notations of ref. [24] we can write,

$$
\rho(t)=\frac{\int_{0}^{1}\left[\theta_{-}(z, t) f_{-}(z)+\theta_{+}(z, t) f_{+}(z)\right] d z}{\int_{0}^{1}\left[f_{-}(z)+f_{+}(z)\right] d z}
$$

in which $z \equiv x / L$ for $x>0$ ( $x$-axis oriented upwards, see Figure 1 ) and $z \equiv-x / L$ for $x<0, f_{-}$and $f_{+}$are functions proportional to the 'efficacy' of a $\gamma$-ray emitted at position $x\left(f_{-}\right.$for $x<0$ in the bottom part of the capillary and $f_{+}$for $x>0$ in the upper part), and $\theta_{-}(z, t)$ and $\theta_{+}(z, t)$ are the relative excesses of tracer concentration in bottom and upper parts of the capillary, respectively, that is

$$
\theta_{i}(z, t) \equiv \frac{\delta C_{T}^{(i)}(z, t)}{C_{T}^{(0)}}
$$

in which $i=+$ or - and $\delta C_{T}^{(i)} \equiv C_{T}^{(i)}-C_{T}^{(0)}, C_{T}^{(-)}$and $C_{T}^{(+)}$are the tracer concentrations for $x<0$ and $x>0$, respectively, and $C_{T}^{(0)}$ is the initial uniform tracer concentration in the capillary. Expressions for the functions $f_{-}$and $f_{+}$were given in ref. [24]. The concentration of the tracer is calculated below by using FD and NM techniques.

\section{Theory}

\subsection{Basic relations in the non-ideal case}

A mixture of two aqueous electrolyte solutions with common anion is considered. It is composed of a major salt, denoted by MA, constituted of monovalent $\mathrm{M}^{+}$and $\mathrm{A}^{-}$ions, and a salt $\mathrm{TA}_{n}\left(\mathrm{~T}^{n+}\right.$ cation with $\left.n \geq 2\right)$ which is present in tracer amount in the solution. The major salt is totally dissociated. On the contrary, the $\mathrm{T}^{n+}$ cation is supposed to form $N$ different complexes of the form $\mathrm{TA}_{k}^{(n-k)+}$, with $k=1, . ., N$, according to the following reactions,

$$
\mathrm{T}^{n+}+k \mathrm{~A}^{-} \rightleftharpoons \mathrm{TA}^{(n-k)+}\left(\beta_{k}\right)
$$


characterized by the thermodynamic cumulative constants $\beta_{k}$, with $k$ going from 1 to $N$.

Hereafter we will adopt the following notations for convenience. We will denote the free cation $\mathrm{T}^{n+}$ with subscript 0 , the first complex $\mathrm{TA}^{(n-1)+}$ with subscript $1, \ldots$, the $k$-th complex $\mathrm{TA}_{k}^{(n-k)+}$ with subscript $k$. The subscripts + and - will be used for the cation and anion of the major salt, $\mathrm{M}^{+}$and $\mathrm{A}^{-}$, respectively.

Moreover, we will make the common assumption that the complexation reactions (eq. (4)) are very fast, so that a local equilibrium prevails for these reactions at any point in the capillary. Then, at any position $x$ and time $t$, one can write for eq. (4),

$$
C_{k}=\beta_{k} \Gamma_{k} C_{0}\left(C_{-}\right)^{k}
$$

in which any $C_{i}$ is the concentration of species $i$ at $x$ and $t\left(C_{i}(x, t)\right)$, and

$$
\Gamma_{k} \equiv \gamma_{0}\left(\gamma_{-}\right)^{k} / \gamma_{k}
$$

where $\gamma_{i}$ stands for the activity coefficient of species $i$ on molarity scale. In the experiments, the quantity of interest is the sum of the concentrations of the various forms of the tracer,

$$
C_{T} \equiv \sum_{k=0}^{N} C_{k}
$$

In this work, besides deviations from ideality due to association itself, we take into account non-ideality effects on the transport of the species and on the formation of the complexes, arising from volume exclusion and electrostatic interactions,. This was done within the framework of the mean spherical approximation (MSA) as detailed below. Now we will write down the main equations governing the transport of the species in the solution.

The diffusion equations obeyed by the concentration $C_{i}$ of a species $i$ is,

$$
\frac{\partial C_{i}}{\partial t}+\nabla J_{i}=Q_{i}
$$

with $t$ the time, $x$ the space coordinate shown in Figure $1, \nabla \equiv \partial / \partial x$ the gradient operator in $1 \mathrm{D}, J_{i}$ the flux density, and $Q_{i}$ the source term coming from the association reaction. In this equation, $i$ denotes a free ion $\mathrm{M}^{+}, \mathrm{A}^{-}$, $\mathrm{T}^{n+}$ or a complex. 
The flux density $J_{i}$ at position $x$ and time $t$ can be derived according to linear transport theory [13] to obtain the well known extended Nernst-Planck equation,

$$
J_{i}=-D_{i} \nabla C_{i}-D_{i} C_{i} \nabla \ln \gamma_{i}+\beta D_{i} C_{i} z_{i} e E
$$

in which $D_{i}$ is the diffusion coefficient of $i, z_{i} e$ is its charge, $\gamma_{i}$ is its activity coefficient, $E$ is the local internal electric field and $\beta=1 / k T$ with $k$ the Boltzmann constant and $T$ the temperature.

The electric field is obtained from the dynamical electroneutrality condition for the major salt MA,

$$
J_{+}=J_{-}
$$

in which the fluxes of the minor species containing $\mathrm{T}$ are neglected ( $\mathrm{T}$ in tracer amounts). At the same time the local electroneutrality condition is fulfilled, which gives

$$
C_{+}=C_{-}=C_{s}
$$

again because one may neglect the concentration of the tracer, so that one gets by combining eqs. (9)-(10),

$$
\mathcal{E} \equiv \beta e E=\frac{D_{+}-D_{-}}{D_{+}+D_{-}} \nabla \ln C_{s}+\frac{1}{D_{+}+D_{-}}\left(D_{+} \frac{\partial \ln \gamma_{+}}{\partial C_{s}}-D_{-} \frac{\partial \ln \gamma_{-}}{\partial C_{s}}\right) \nabla C_{s}
$$

for which we used eq. (11) and $\mathcal{E}$ has the dimension of the inverse of a length.

The combination of eqs. (9)-(12) yields the well-known relation [26, 27],

$$
J_{s}=-D_{s}\left(1+\frac{\partial \ln \gamma_{s}}{\partial \ln C_{s}}\right) \nabla C_{s}
$$

for the flux of the major salt $s \equiv$ MA, with $D_{s}$ the Nernst-Hartley diffusion coefficient of the salt,

$$
D_{s} \equiv \frac{2 D_{+} D_{-}}{D_{+}+D_{-}}
$$

which is comprised between $D_{-}$and $D_{+}$. Eq. (13) shows that the effect of non-ideality is to modify locally the diffusion coefficient of the salt by a factor of $\left(1+\partial \ln \gamma_{s} / \partial \ln C_{s}\right)$.

The diffusion equation for the major salt is readily obtained from eq. (8) with $Q_{+}=Q_{-}=0$ and eqs. (10), (11), (13),

$$
\frac{\partial C_{s}}{\partial t}=D_{s} \nabla\left[\left(1+\frac{\partial \ln \gamma_{s}}{\partial \ln C_{s}}\right) \nabla C_{s}\right]
$$


For the minor species containing the tracer we get using eqs. (8) and (9),

$$
\frac{\partial C_{i}}{\partial t}=D_{i} \Delta C_{i}+D_{i} \nabla\left[C_{i}\left(\nabla \ln \gamma_{i}-z_{i} \mathcal{E}\right)\right]+Q_{i}
$$

in which $\Delta \equiv \nabla \cdot \nabla=\partial^{2} / \partial x^{2}$ is the Laplace operator in $1 \mathrm{D}$.

Non ideality was described using the mean spherical approximation (MSA) [28], with values for the ion sizes and the solution permittivity that did not vary with salt concentration. A specific size was attributed to each complex (see the Results and Discussion section). The activity coefficients of the species were calculated at the McMillan-Mayer level (solvent of constant chemical potential and regarded as a continuum) and were converted to the experimental (Lewis-Randall) level by using the formula [28, 29, 30],

$$
\ln \gamma_{i}=\ln \gamma_{i}^{M M}-2 C_{s} \Phi^{M M} V_{i}
$$

with $\gamma_{i}^{M M}$ and $\Phi^{M M}$ the activity and osmotic coefficients, respectively, calculated at the McMillan-Mayer (MM) level within the MSA model, and $V_{i}$ the partial molar volume (PMV) of $i$. The PMVs of $\mathrm{Zn}^{2+}$ and $\mathrm{Cl}^{-}$ions were estimated by taking the volume of the ion [31], which gives values of 0.001 and $0.012 \mathrm{~L} \mathrm{~mol}^{-1}$, respectively. Indeed it was found that this simple choice gives the good order of magnitude for the PMV of $\mathrm{ZnCl}_{2}$ at infinite dilution $\left(0.0235 \mathrm{~L} \mathrm{~mol}^{-1}\right)$. The PMVs of the complexes were computed from the formula, $V_{k}=V_{Z n^{2+}}+k V_{C l^{-}}$.

Since the tracer is in very small amount in the solution, the activity coefficients of the complexes and of the salt ions only depend on the local concentration of the salt. Thus, in what follows, all physical quantities only depend on the local value of $C_{s}$. Consequently the gradient of any quantity $f$ may be calculated as,

$$
\nabla f=f^{\prime} \times \nabla C_{s}
$$

in which $f^{\prime}$ is the derivative of $f$ w.r.t. $C_{s}, f^{\prime} \equiv \partial f / \partial C_{s}$.

The diffusion equation for total tracer may be obtained from the equations for the various forms of the tracer. By considering that the concentrations of the complexes are equilibrated at any point in the capillary, we obtain by utilizing eqs. (5), (7) and (11),

$$
C_{k}=\beta_{k} \Gamma_{k} C_{s}{ }^{k} C_{T} / \sum_{n=0}^{N} \beta_{n} \Gamma_{n} C_{s}{ }^{n}
$$


In this relation we make the usual convention for $n=0$, namely that $\beta_{0}=1$. We note that equilibration, expressed by eq. (19), is made very simple by the fact that $\mathrm{T}$ is in tracer amounts in the experiments.

Summation of the diffusion equations for the free and associated forms of the tracer, together with the use of eqs. (12) and (19) in eq. (16), and also $\sum_{i} Q_{i}=0$, leads to the diffusion equation for total tracer. After some algebra one gets,

$$
\frac{\partial C_{T}}{\partial t}=D_{T} \Delta C_{T}+d_{1}\left(\frac{\nabla C_{s}}{C_{s}}\right) \nabla C_{T}+d_{2}\left(\frac{\nabla C_{s}}{C_{s}}\right)^{2} C_{T}+d_{s}\left(\frac{\Delta C_{s}}{C_{s}}\right) C_{T}
$$

in which

$$
D_{T}=\frac{\sum_{k=0}^{N} D_{k} \beta_{k} \Gamma_{k} C_{s}{ }^{k}}{\sum_{k=0}^{N} \beta_{k} \Gamma_{k} C_{s}{ }^{k}}
$$

is the (local) mean diffusion coefficient of the tracer, and the parameters $d_{1}$, $d_{2}$ and $d_{s}$ have the dimension of a diffusion coefficient (but may be negative). Their expression is given in the Appendix.

\subsection{Normal-mode treatment}

The normal-mode (NM) treatment consists of linearizing the transport equations. Any physical quantity, $q(x, t)$, is decomposed in two terms: an equilibrium term, $q^{\infty}$ (for $t \rightarrow \infty$ ), and a non-equilibrium or perturbation term $\delta q(x, t)$, that is, $q(x, t)=q^{\infty}+\delta q(x, t)$. Linearization is implemented by keeping only the first-order terms in the equations.

Here, the concentrations of total tracer, $C_{T}(x, t)$, and of the salt, $C_{s}(x, t)$ are expressed as, $C_{i}(x, t)=C_{i}^{\infty}+\delta C_{i}(x, t)$, in which $C_{T}^{\infty}=C_{T}^{(0)}$ and $C_{s}^{\infty}=$ $C_{s}^{(0)} / 2$ (with $C_{s}^{(0)}$ the initial salt concentration in the bottom half of the capillary). The diffusion coefficients appearing in eq. (20) may be written as, $D=D^{\infty}+\delta C_{s} D^{\prime}$ (with $D=D_{T}, d_{1}, d_{2}, d_{s}$ ). Linearizing eq. (20) w.r.t. $\delta C_{T}$ and $\delta C_{s}$ leads to a diffusion equation for the total tracer in which the terms proportional to $d_{1}$ and $d_{2}$ disappear because they are of second order, $\delta C_{s} \delta C_{T}$ or $\left(\delta C_{s}\right)^{2}$, respectively (because $\nabla C_{i}=\nabla \delta C_{i}$ for $i=s, T$ ). So one obtains straightforwardly,

$$
\frac{\partial \delta C_{T}}{\partial t}=D_{T}^{\infty} \Delta \delta C_{T}+d_{s}^{\infty} \frac{C_{T}^{\infty}}{C_{s}^{\infty}} \Delta \delta C_{s}
$$


in which $D_{T}^{\infty}$ and $d_{s}^{\infty}$ are the values of $D_{T}$ and $d_{s}$ (eqs. (21) and (32)) at equilibrium, for $C_{s}=C_{s}^{\infty}$.

Analogously, the linearized diffusion equation for the salt is,

$$
\frac{\partial \delta C_{s}}{\partial t}=D_{s}^{\infty} \Delta \delta C_{s}
$$

in which

$$
D_{s}^{\infty} \equiv D_{s}\left(1+\left.\frac{\partial \ln \gamma_{s}}{\partial \ln C_{s}}\right|_{C_{s}=C_{s}^{\infty}}\right)
$$

is the effective salt diffusivity at equilibrium.

The coupled equations, eqs. (22) and (23), can be solved by using Fourier transform on space (denoted by an asterisk) and Laplace transform on time (denoted by a tilde), as exposed in previous work [11]. The result is,

$$
\delta \tilde{C}_{T}^{*}(q, s)=\delta \tilde{C}_{T}^{*(s)}(q, s)+\delta \tilde{C}_{T}^{*(m)}(q, s)
$$

with

$$
\begin{gathered}
\delta \tilde{C}_{T}^{*(s)}(q, s) \equiv \frac{\delta C_{T}^{*}(q, t=0)}{s+q^{2} D_{T}^{*}} \\
\delta \tilde{C}_{T}^{*(m)}(q, s) \equiv-d_{s}^{\infty} \frac{C_{T}^{\infty}}{C_{s}^{\infty}} q^{2} \frac{\delta C_{s}^{*}(q, t=0)}{\left(s+q^{2} D_{T}^{\infty}\right)\left(s+q^{2} D_{s}^{\infty}\right)}
\end{gathered}
$$

in which $q$ and $s$ are the Fourier and Laplace variables associated to $x$ and $t$, respectively, and $\delta C_{T}^{*(s)}$ is the self-diffusion term and $\delta C_{T}^{*(m)}$ is the migration term for the tracer.

We note that, in the present experiments, the initial tracer concentration profile is uniform (equal concentration in the two halves of the capillary), so that $\delta C_{T}(x, t=0)=0$ and thus $\delta C_{T}^{*}(q, t=0)=0$. Therefore, only the migration term, $\delta \tilde{C}_{T}^{*(m)}$, will remain in Eq. (25).

Finally, by using the same method as in previous work [11], we find that the solution for the function $\rho(t)$ (Eq. (1) or (2)) is,

$$
\rho_{N M}(t)=-\frac{4}{\pi} d_{s}^{\infty} \sum_{k=0}^{\infty} \frac{F_{k}}{2 k+1} \frac{\exp \left(-q_{2 k+1}^{2} D_{T}^{\infty} t\right)-\exp \left(-q_{2 k+1}^{2} D_{s}^{\infty} t\right)}{D_{s}^{\infty}-D_{T}^{\infty}}
$$

where $q_{n} \equiv n \pi / 2 L$, and

$$
F_{k}=\int_{0}^{1}\left[f_{-}(z)-f_{+}(z)\right] \sin \left[(2 k+1) \frac{\pi}{2} z\right] d z / \int_{0}^{1}\left[f_{-}(z)+f_{+}(z)\right] d z
$$


In eq. (28) $d_{s}^{\infty}$ was computed from eq. (32) at equilibrium and the quantity $\Psi_{k}^{\prime}$ (appearing in eq. 32 for $d_{s}$ ) was calculated by numerical differentiation.

For the geometry of the set-up depicted in Figure 1, the coefficients $F_{k}$ were found to have the following values [24]: $F_{0}=0.472677, F_{1}=0.0564312$, $F_{2}=0.0233391, F_{3}=0.00424871, F_{4}=0.004660106, F_{5}=0.0000347272$. Since all these parameters are positive, we deduce from eq. (28) that $\rho_{N M}$ has the sign of $-d_{s}^{\infty}$.

For sufficiently long times, eq. (28) may be reduced to its first exponential term. In the present case we find that this approximation is valid to $1 \%$ accuracy for $t \gtrsim 10 \mathrm{~h}$. By differentiation of this simplified expression w.r.t. time one gets the order of magnitude of the time $t_{m}$ at which $\rho_{N M}$ is maximum (in absolute value),

$$
t_{m}=\frac{4 L^{2}}{\pi^{2} D_{s}^{\infty}} \frac{\ln x}{x-1}
$$

with $x \equiv D_{T}^{\infty} / D_{s}^{\infty}$. By inserting this expression for $t_{m}$ into eq. (28) we find after some simplifications that the maximum amplitude of $\rho_{N M}\left(\right.$ at $\left.t=t_{m}\right)$ is approximately given by,

$$
\rho_{N M}\left(t=t_{m}\right) \simeq-\frac{4}{\pi} F_{0} \frac{d_{s}^{\infty}}{D_{s}^{\infty}} x^{x /(1-x)}
$$

The function $x^{x /(1-x)}$ decreases monotonously with $x$ from a value of 1 for $x=0$ to 0 for large values of $x$.

\subsection{Finite difference method}

A classic finite difference algorithm was used in this work. The main ingredients of the method were described in our previous study [11]. The diffusion equations were discretized in space and time, and the system was symmetrized at both ends of the capillary. A number of $N_{c}=200$ cells was taken to represent the capillary. The result was observed to be nearly independent of $N_{c}$ for $N_{c} \geq 200$. At each time step, all species were let to diffuse individually according to eqs. (15) and (16) with $Q_{i}=0$, and then the concentrations of the complexes were equilibrated in each cell.

A difference in the present work comes from the inclusion of non-ideality. In our earlier treatment [11], conservation of matter in the capillary was ensured by imposing a zero electric field at both ends of the capillary. In the present work, it was found that this condition could be satisfied by discretizing eq. (16) (without expanding it) and by imposing the relation, $\nabla \ln \gamma_{i}-z_{i} \mathcal{E}=0$, in cells no. 1 and $N_{c}$ at every time step. 


\section{Results and Discussion}

\subsection{Parameter values}

First, parameter values had to be determined for the description of association, activity coefficients and transport of species in the model. An approximate procedure had to be used in order to reduce the number of parameters in the model. Their values are collected in Table 1. They were determined as follows.

The diameters of the salt ions $\mathrm{Li}^{+}$and $\mathrm{Cl}^{-}$were required for the computation of activity coefficients of all species within the MSA [30]. In this framework, we usually keep the diameter of $\mathrm{Cl}^{-}$ion equal to its Pauling crystallographic value $(3.62 \AA)$ because it is essentially unhydrated. Then, the diameter of (hydrated) $\mathrm{Li}^{+}$ion was adjusted by fitting the experimental osmotic coefficient of aqueous $\mathrm{LiCl}$ solution up to $2 \mathrm{M}$, by keeping the dielectric constant of solution equal to that of water.

The values for the cumulative constants $\beta_{n}$ were taken from a database published in the geochemical community for the determination of speciation in natural waters [32]. These values are close to those proposed by Marcus [33].

A fit of experimental osmotic coefficient data for zinc perchlorate solution up to $0.2 \mathrm{~mol} \mathrm{~kg}^{-1}$ [34] within the MSA with constant solution permittivity gave an optimum zinc(II) ion diameter of $\sim 6.4 \AA$ with a diameter of 4.53 $\AA$ for the perchlorate ion [30]. In this fit, zinc perchlorate was regarded as being not associated as found experimentally [35, 36]. This value of $6.4 \AA$ was taken here for the computation of the activity coefficient of free zinc(II) ion in $\mathrm{LiCl}$ solution.

It is known from experiment that the structure of the zinc(II) ion in water is a hexa-coordinated aquo-complex, $\mathrm{ZnW}_{6}^{2+}[36]$ (in which $\mathrm{W}$ represents the water molecule), with an average lifetime of a few nanoseconds [37]. The maximum diameter of this species is of the order of $1.76+2 \times 2.76 \sim 7.3 \AA$, as found by adding the diameters of the bare zinc(II) ion [31] and two times that of the water molecule. Besides, the diameter of a sphere having the same volume as this species is $\sim 5.1 \AA$. We note that the MSA adjusted diameter of the zinc(II) ion in perchlorate solution is nearly the average of these two values. So, the diameter of $6.4 \AA$ may be interpreted as the effective size of the aquo-complex in the MSA.

The structure of zinc chloride complexes in water has been studied experimentally [38] and by ab initio numerical simulation [21, 22, 39]. The consis- 
tent picture emerging from these studies is one in which the monochloro and the dichloro complexes are hexa-coordinated, and the trichloro and tetrachloro complexes are tetra-coordinated. Their formulas are $\mathrm{ZnClW}_{5}^{+}$, $\mathrm{ZnCl}_{2} \mathrm{~W}_{4}^{0}, \mathrm{ZnCl}_{3} \mathrm{~W}^{-}$and $\mathrm{ZnCl}_{4}^{2-}$. The change of coordination in the complexes with 3 and 4 chlorides is likely to originate from the fact that it lowers the electrostatic repulsion between the charges on the chlorides as compared to hexa-coordinated compounds [39]. The diameters taken for the complexes in the MSA model were estimated from the MSA diameter of zinc(II) ion $\left(\sigma_{0}=6.4 \AA\right)$ and the volumes of the complexes $\left(v_{k}\right)$ by using the formula, $\sigma_{k}=\sigma_{0}\left(v_{k} / v_{0}\right)^{1 / 3}$. This led to the values listed in Table 1.

The diffusivities of $\mathrm{Li}^{+}, \mathrm{Cl}^{-}$and $\mathrm{Zn}^{2+}$ at $25^{\circ} \mathrm{C}$ were obtained from the values for the limiting equivalent conductivities [27] by using a classic conversion formula (eq. 11.49 of ref. [27]). Those for the complexes were assessed by utilizing the $D$ value for the zinc(II) ion, the values of the complex diameters $\left(\sigma_{k}\right)$, and the Stokes-Einstein formula, from which we got, $D_{k}=D_{0} \sigma_{0} / \sigma_{k}$, by assuming that $\sigma_{k}$ is a good approximation for the hydrodynamic diameter of the complex $k$. It is noticed in Table 1 that the value of $D$ is not monotonous in the series because of the transition from hexa- to tetra-coordination when passing from $\mathrm{ZnCl}_{2}$ to $\mathrm{ZnCl}_{3}^{-}$.

Table 1: Values taken for diameters $\left(\sigma_{i}\right)$, diffusivities $\left(D_{i}\right)$, and cumulative formation constants $\beta_{i}$ of species.

\begin{tabular}{lccc}
\hline Species & $\sigma_{i}(\AA)$ & $D_{i}\left(10^{-5} \mathrm{~cm}^{2} \mathrm{~s}^{-1}\right)$ & $\log _{10} \beta_{i}$ \\
\hline $\mathrm{Li}^{+}$ & 4.26 & $1.03^{a}$ & \\
$\mathrm{Cl}^{-}$ & 3.62 & $2.03^{a}$ & \\
$\mathrm{ZnW}_{6}{ }^{2+}$ & 6.40 & $0.703^{a}$ & \\
$\mathrm{ZnClW}_{5}{ }^{+}$ & 6.65 & 0.677 & 0.49 \\
$\mathrm{ZnCl}_{2} \mathrm{~W}_{4}{ }^{0}$ & 6.88 & 0.654 & 0.62 \\
$\mathrm{ZnCl}_{3} \mathrm{~W}^{-}$ & 6.50 & 0.692 & 0.51 \\
$\mathrm{ZnCl}_{4}^{2-}$ & 6.75 & 0.667 & 0.20 \\
\hline
\end{tabular}

\subsection{Forces in play}

In order to gain more insight into the process, the speciation found for the zinc ion at $\mathrm{LiCl}$ concentrations of $0.5 \mathrm{M}, 1 \mathrm{M}$ and $2 \mathrm{M}$ from the MSA description, and in the ideal case with association is shown in Table 2. The first two concentrations, $0.5 \mathrm{M}$ and $1 \mathrm{M}$, correspond to the values for the concentration 
of $\mathrm{LiCl}$ at point $\mathrm{O}$ (see Figure 1) when the bottom of the capillary is filled with $1 \mathrm{M}$ or $2 \mathrm{M} \mathrm{LiCl}$ solution at $t=0$. Indeed the $\mathrm{LiCl}$ concentration at point $\mathrm{O}$ is approximately constant in time during the diffusion process and it is equal to half of the initial concentration in the bottom part of the capillary.

Table 2: Speciation and average valency for zinc at various concentrations of LiCl, obtained from the present MSA model (first line for every $\mathrm{LiCl}$ concentration) and in the ideal case (second line, in parentheses).

\begin{tabular}{ccccccc}
\hline$C_{L i C l}$ & $\mathrm{Zn}^{2+}$ & $\mathrm{ZnCl}^{+}$ & $\mathrm{ZnCl}_{2}$ & $\mathrm{ZnCl}_{3}^{-}$ & $\mathrm{ZnCl}_{4}^{2-}$ & $\langle z\rangle^{a}$ \\
\hline $0.5 \mathrm{M}$ & $25.4 \%$ & $40.8 \%$ & $19.3 \%$ & $11.6 \%$ & $2.9 \%$ & 0.74 \\
& $(23.2 \%)$ & $(37.3 \%)$ & $(26.2 \%)$ & $(10.6 \%)$ & $(2.7 \%)$ & $(0.68)$ \\
$1 \mathrm{M}$ & $10.5 \%$ & $24.0 \%$ & $19.8 \%$ & $27.7 \%$ & $18.0 \%$ & -0.19 \\
& $(7.3 \%)$ & $(23.1 \%)$ & $(31.8 \%)$ & $(25.2 \%)$ & $(12.6 \%)$ & $(-0.13)$ \\
$2 \mathrm{M}$ & $2.4 \%$ & $4.4 \%$ & $6.5 \%$ & $22.2 \%$ & $64.5 \%$ & -1.4 \\
& $(1.3 \%)$ & $(8.1 \%)$ & $(22 \%)$ & $(34.5 \%)$ & $(34.1 \%)$ & $(-0.92)$ \\
\hline
\end{tabular}

${ }^{a}$ Average valency of zinc species in its various forms

It must be noticed that this zone of the capillary is where most of the internal electric field effect is created. This is due to the fact that the electric field is the most intense in this region of highest gradient of $\mathrm{LiCl}$. This field originates from the diffusion of the major ions. The chloride ion diffusivity being larger than that of the lithium ion, an electric field is created, directed from the bottom to the top of the capillary, that pulls the slow ion (the positive lithium ion) and slows down the fast ion (the chloride ion).

It is seen in this table that as expected the speciation of zinc is shifted towards the formation of complexes of higher stoichiometry when $C_{\mathrm{LiCl}}$ is increased. In the upper region of the capillary, as long as $\mathrm{LiCl}$ has not reached this zone, the zinc is in the form of $\mathrm{Zn}^{2+}$ ion (dilute $\mathrm{ZnCl}_{2}$ solution). It is seen also in this table that the effect of deviations from ideality is rather weak at $0.5 \mathrm{M} \mathrm{LiCl}$, moderate at $1 \mathrm{M}$ and appreciable at $2 \mathrm{M}$.

The value of the average valency of zinc containing species, $\langle z\rangle$, is given in the last column of Table 2. It has a value of 2 at very low $C_{L i C l}$, at which the free zinc(II) ion is the dominant form of zinc. This quantity gives an indication of the net effect of the electric field on the zinc species (taken globally, in all forms) because the complexes are expected to have similar diffusivities. In particular, since $\langle z\rangle$ is positive at $0.5 \mathrm{M} \mathrm{LiCl}$ and negative at $1 \mathrm{M}$, we find that the electric field should have opposite effects on zinc at these two concentrations (which correspond to those at point $\mathrm{O}$ in the capillary 
for initial $1 \mathrm{M}$ and $2 \mathrm{M} \mathrm{LiCl}$, respectively, in the bottom part). At very low $\mathrm{LiCl}$ concentration, the zinc(II) ion would be dragged in the direction of the electric field (from the bottom to the top of the capillary) because it is positive, which would make the function $\rho$ take negative values. When $C_{\mathrm{LiCl}}$ is increased, $\langle z\rangle$ is also decreased. Then we expect that $\rho$ will take less negative values for initial $2 \mathrm{M} \mathrm{LiCl}$ than for $1 \mathrm{M}$ under the effect of the electric field.

Besides the influence of the electric field, the second main phenomenon governing the motion of zinc is self-diffusion (the term $-D_{i} \nabla C_{i}$ in eq. (9)). In solutions of $1 \mathrm{M}$ and $2 \mathrm{M} \mathrm{LiCl}$ initially in the bottom of the capillary, the $\mathrm{Zn}^{2+}$ ion is the minor zinc species and it is by far the major ion in the top part. This strong gradient causes natural diffusion of $\mathrm{Zn}^{2+}$ ion from the top to the bottom half of the capillary. It is the reverse situation as regards the complexes that are nearly inexistent in the top part. This induces crossed fluxes of free zinc(II) ion and of the complexes. However the overall flux of zinc from the bottom to the top of the capillary is likely to be small because these fluxes may compensate each other.

The last effect is that of deviations from ideality on the fluxes as expressed in eq. (9) by the term $-D_{i} C_{i} \nabla \ln \gamma_{i}$. The activity coefficients of the zinc compounds obtained from the MSA model are collected in Table 3. The bivalent ions $\mathrm{Zn}^{2+}$ and $\mathrm{ZnCl}_{4}^{2-}$ have the lower values because they have higher electrostatic interactions with the major salt, which gives them lower electrostatic energies. The monovalent ions $\mathrm{ZnCl}^{+}$and $\mathrm{ZnCl}_{3}^{-}$, and still more the neutral ion $\mathrm{ZnCl}_{2}$, have higher activity coefficient values.

Table 3: Values of $\ln \gamma_{i}$ for the zinc compounds obtained from the present model.

\begin{tabular}{cccccc}
\hline$[\mathrm{LiCl}]$ & $\mathrm{Zn}^{2+}$ & $\mathrm{ZnCl}^{+}$ & $\mathrm{ZnCl}_{2}$ & $\mathrm{ZnCl}_{3}^{-}$ & $\mathrm{ZnCl}_{4}^{2-}$ \\
\hline $1 \mathrm{M}$ & -0.919 & 0.372 & 0.773 & 0.412 & -0.951 \\
$2 \mathrm{M}$ & -0.493 & 1.24 & 1.86 & 1.08 & -0.394 \\
\hline
\end{tabular}

According to eq. (9) the flux of species $i$ due to non-ideality has the sign of $-\nabla \ln \gamma_{i}$. Thus, with the $x$-axis being oriented from the bottom to the top of the capillary (see Figure 1), the sign of the overall flux for a species $i$ may be expected to have basically the sign of $\ln \gamma_{i}$ reported in Table 3. Consequently, according to this table, we see that the species should behave differently with respect to this effect. On the other hand, we observe that the values of $\ln \gamma_{i}$ all increase in algebraic value from $1 \mathrm{M}$ to $2 \mathrm{M} \mathrm{LiCl}$ concentration. Therefore 
the effect of activity coefficients should be stronger at $2 \mathrm{M} \mathrm{LiCl}$ than at $1 \mathrm{M}$. The transfer of zinc under this effect from the bottom to the top of the capillary should be larger at $2 \mathrm{M}$ than at $1 \mathrm{M}$, so making $\rho$ more negative.

This preliminary discussion shows that the transport of zinc in the capillary is the result of several antagonistic effects. At low $C_{\mathrm{LiCl}}$, the main effect would be due to the electric field (which remains finite by virtue of eq. (12) and becomes independent of $C_{s}$ when $C_{s}$ becomes very small), so that $\rho$ would be negative. When $C_{L i C l}$ is increased, the effect of self-diffusion must be small, that of the electric field is to reduce the amplitude of $\rho$, and that of activity coefficients is to enhance it. The theoretical FD and NM treatments were utilized to solve this problem. The results are exposed in the next section.

\subsection{Comparison of experimental and theoretical results}

Typical experimental curves (denoted by the letter "e") and theoretical results are presented in Figures 2 and 3, in which $-\rho$ is plotted as a function of time. The fact that the experimental plots appear as broken lines originates from the natural fluctuations in $\gamma$-emission by the radioactive zinc tracer.

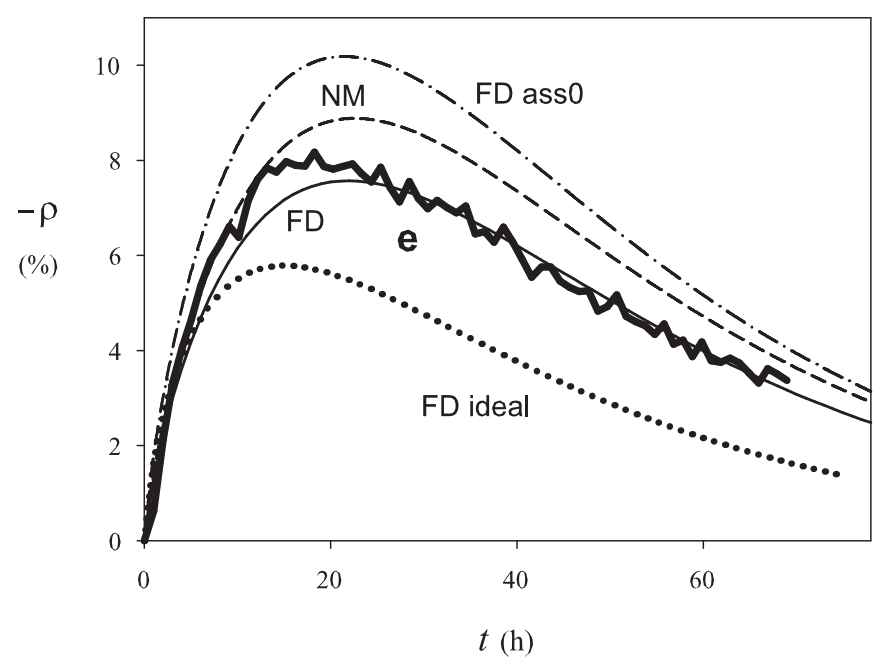

Figure 2: Plot of $-\rho$ as a function of time for the experiment with $1 \mathrm{M} \mathrm{LiCl}$. (e) Experimental points; (FD) and (NM) FD and NM results with association and deviations from ideality, respectively; (FD ass0) FD without association but with deviations from ideality; (FD ideal) FD for ideal solution with association. 


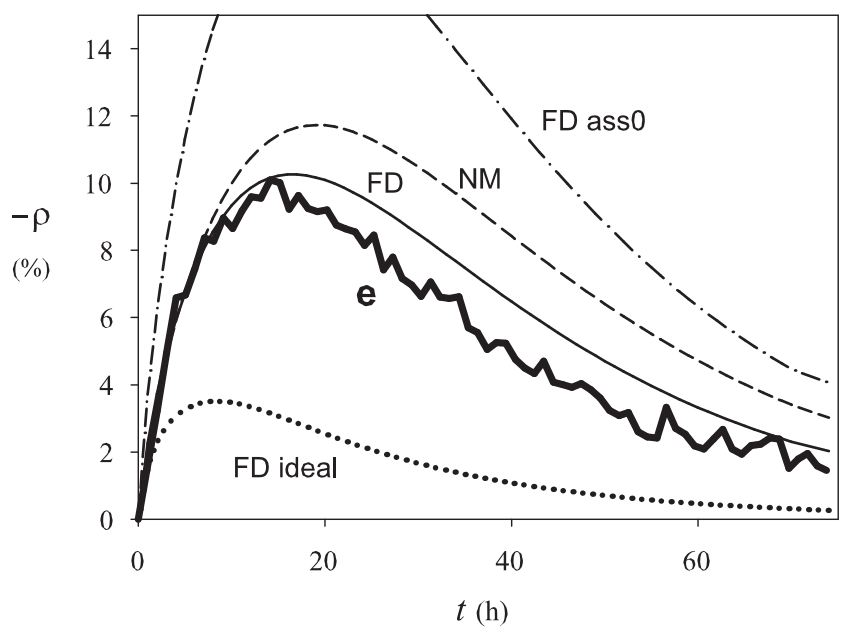

Figure 3: Plot of $-\rho$ as a function of time for the experiment with $2 \mathrm{M} \mathrm{LiCl}$. (e) Experimental points; (FD) and (NM) FD and NM results with association and deviations from ideality, respectively; (FD ass0) FD without association but with deviations from ideality; (FD ideal) FD for ideal solution with association.

In Figure 2, the FD result including association and deviations from ideality is in very good agreement with the experimental data. The FD curves obtained in the ideal case (FD ideal), and without association (FD ass0), are also shown. They indicate how sensitive the FD result is to the incorporation of these two phenomena. It is noticed that the ideal plot for $-\rho$ is below the non-ideal result (maxima of ca. 6\% and $8 \%$, respectively). On the contrary, in the absence of association, the result (FD ass0) is significantly above the FD plot, mainly because associated forms of zinc ion have a lower (possibly negative) electric charge than the free zinc(II) ion. Consequently the effect of the electric field is lower on these species or it is in the reverse direction. The normal modes (NM) plot is slightly above the FD plot, with a relative difference of $\sim 17 \%$ between the two maxima. Moreover, the positions in time of the maxima for $-\rho$ are nearly identical $(\sim 22.0 \mathrm{~h}$ and $22.6 \mathrm{~h}$ for FD and NM, respectively), and the value for NM coincides with the one given by eq. $(30)\left(t_{m}=22.6 \mathrm{~h}\right)$. The value for the NM maximum is accurately given by eq. (31).

In Figure 3, the same curves as in Figure 2 are plotted in the case of initial $2 \mathrm{M} \mathrm{LiCl}$. It is observed that the full FD result (accounting for association 
and activity coefficients) is close to the experimental curve ("e"). At the same time, the sensitivity of the FD result to the inclusion of association and deviations from ideality is much more pronounced than at $1 \mathrm{M} \mathrm{LiCl}$ (see Figure 2). Here, the FD result in the ideal case is much below the FD plot, which shows the importance of including non-ideality in the description. Moreover, the formation of zinc complexes is seen to cause a large drop in the observed maximum for $-\rho$. In the absence of association we find that the maximum for the FD plot is of $\sim 18 \%$ as compared to $\sim 10 \%$ for the experiment and the full FD curve. Thus, the full FD calculation including association and activity coefficients seems capable of capturing the main effects acting on the various forms of the solute. Besides, the NM plot is just a bit higher than the FD plot, with a relative difference between the two maxima of $\sim 14 \%$. The positions in time of the maxima are a bit more different from each other than at $1 \mathrm{M} \mathrm{LiCl}(\sim 17 \mathrm{~h}$ and $19 \mathrm{~h}$ for FD and NM, respectively). This value for NM is precisely given by eq. $(30)\left(t_{m}=19.2 \mathrm{~h}\right)$.

\section{Conclusion}

It has been found that the model developed in this work provides a good prediction of transient diffusion profiles for a multiply associating ion forming chemical complexes in a supporting electrolyte. This satisfactory description was developed by taking literature values for the association constants and by describing deviations from ideality within the MSA with reasonable input parameter values. The prediction consisted of using a finite difference algorithm and, besides, an approximate normal mode analytic calculation. Both accounted for diffusion, internal electric field and activity coefficients for all species. The normal mode result was found to be a little larger than the more accurate finite difference result. The NM method may be used to estimate the order of magnitude of the experimental effect and it provides a confirmation of the FD result.

In subsequent work, we will study the case of electrolytes forming several ionic complexes. In such systems, the cation interacts strongly with an anion through electrostatic forces but does not form covalent complexes. This is for instance the case of lanthanides with some halides. It will be interesting to examine whether coupling diffusion can be described within the classic chemical model [40] in which an ionic complex is regarded as a true chemical complex. 


\section{Appendix}

The coefficients appearing in eq. (20) are as follows,

$$
\begin{aligned}
& d_{1}=\sum_{k=0}^{N} D_{k} \beta_{k} C_{s}{ }^{k}\left[\left(2 k+\varphi_{k}\right) \Psi_{k}+2 C_{s} \Psi_{k}^{\prime}\right] \\
& d_{2}=\sum_{k=0}^{N} D_{k} \beta_{k} C_{s}{ }^{k}\left\{\left[(k-1)\left(k+\varphi_{k}\right)+C_{s} \varphi_{k}^{\prime}\right] \Psi_{k}+C_{s}\left(2 k+\varphi_{k}\right) \Psi_{k}^{\prime}+C_{s}{ }^{2} \Psi_{k}^{\prime \prime}\right\} \\
& d_{s}=\sum_{k=0}^{N} D_{k} \beta_{k} C_{s}{ }^{k}\left[\left(k+\varphi_{k}\right) \Psi_{k}+C_{s} \Psi_{k}^{\prime}\right]
\end{aligned}
$$

where a prime denotes differentiation w.r.t. $C_{s}$, i.e. $f^{\prime} \equiv \partial f / \partial C_{s}$, and we have introduced the following definitions

$$
\begin{aligned}
\varphi_{k} & \equiv \frac{\partial \ln \gamma_{k}}{\partial \ln C_{S}}-\frac{z_{k}}{D_{+}+D_{-}}\left[D_{+}\left(1+\frac{\partial \ln \gamma_{+}}{\partial \ln C_{S}}\right)-D_{-}\left(1+\frac{\partial \ln \gamma_{-}}{\partial \ln C_{S}}\right)\right] \\
\Psi_{k} & \equiv \Gamma_{k} / \sum_{n=0}^{N} \beta_{n} \Gamma_{n} C_{s}{ }^{n}
\end{aligned}
$$

Note that, in these relations, $\beta_{k} C_{s}{ }^{k}, \Gamma_{k}, \varphi_{k}$ and $\Psi_{k}$ are quantities without dimension.

The derivatives in these equations involve differentiation of individual activity coefficients w.r.t. the salt concentration $C_{s}$. They may be calculated for a given model of deviations from ideality, either analytically (if possible) or numerically.

\section{Ackowledgement}

This work was supported by a grant from ECOS-Nord (project M11P03).

\section{References}

[1] H.J.V. Tyrrell, K.R. Harris, Diffusion in Liquids, Butterworths, London, 1984. 
[2] R. Mills, A. Perera, J.P. Simonin, L. Orcil, P. Turq, Coupling of diffusion Processes in Multicomponent Electrolyte Solutions, J. Phys. Chem. 89, 2722-2725 (1985).

[3] S.B. Yaakov, Diffusion of seawater ions. I. Diffusion of seawater into a dilute solution. Geochim. Cosmochim. Acta. 36, 1395-1407 (1972).

[4] A.C. Lasaga, The treatment of multi-component diffusion and ion pairs in diagenetic fluxes, Am. J. Sci. 279, 324-346 (1979).

[5] B. Eisenberg, Ionic channels in biological membranes - electrostatic analysis of a natural nanotube, Contemp. Phys. 39, 447-466 (1998).

[6] A.M. Simon, P. Doran, R. Paterson, Assessment of diffusion coupling effects in membrane separation. Part I. Network thermodynamics modelling, J. Memb. Sci., 109, 2, 231-246 (1996).

[7] E. Samson, J. Marchand, Calculation of ionic diffusion coefficients on the basis of migration test results, Mater. Struct. 36, 156-165 (2003).

[8] J. Bai, Advanced Fibre-Reinforced Polymer (FRP) Composites for Structural Applications, Woodhead Publishing Series in Civil and Structural Engineering, Elsevier Science, 2013.

[9] B.P. Boudreau, F.J.R. Meysmanb, J.J. Middelburg, Multicomponent ionic diffusion in porewaters: Coulombic effects revisited, Earth Planet. Sc. Lett. 222, 653666 (2004).

[10] D.G. Miller, Application of Irreversible Thermodynamics to Electrolyte Solutions. I. Determination of Ionic Transport Coefficients $l_{i j}$ for Isothermal Vector Transport Processes in Binary Electrolyte Systems, J. Phys. Chem. 70, 2639-2659 (1966).

[11] J.P. Simonin, J.F. Gaillard, P. Turq, E. Soualhia, Diffusion coupling in electrolyte solutions. 1. Transient effects on a tracer ion: sulfate, J. Phys. Chem. 92, 1696-1700 (1988).

[12] J.P. Simonin, P. Turq, E. Soualhia, G.Michard, J.F. Gaillard, Transport coupling of ions: Influence of ion pairing and $\mathrm{pH}$ gradient Application to the study of diagenetic fluxes, Chem. Geol. 78, 343-356 (1989). 
[13] P. Turq, L. Orcil, M. Chemla, Normal Mode Analysis od Diffusion in Multicomponent Electrolyte Solutions. 1. Nonassociating Electrolytes, J. Phys. Chem. 86, 4062-4067 (1982).

[14] Free software developed in Sweden at KTH (Royal Institute of Technology), http://www2.lwr.kth.se/English/OurSoftware/vminteq/.

[15] Software developed in the US, http://www.mineql.com/index.html.

[16] Software developed at Ecoles des Mines in France, http://www.geosciences.mines-paristech.fr/en/training/continuingeducation/chess-training.

[17] Free software developed at US Geological Survey, http://wwwbrr.cr.usgs.gov/projects/GWC_coupled/phreeqc/.

[18] Free software developed by Wilko Verweij in Netherlands, http://www.cheaqs.eu/.

[19] L.G. Sillen, A.E. Martell, Stability Constants of Metal-Ion Complexes, 2nd edition, The Chemical Society, London (1964).

[20] NIST Database 46 Version 8.0, NIST Critically Selected Stability Constants of Metal Complexes, http://www.nist.gov/srd/nist46.cfm.

[21] O.G. Parchment, M.A. Vincent, I.H. Hillier, Speciation in Aqueous Zinc Chloride. An ab Initio Hybrid Microsolvation/Continuum Approach, J. Phys. Chem. 100, 9689-9693 (1996).

[22] C.C. Pye, C.M. Corbeil, W.W. Rudolph, An ab initio Investigation of Zinc Chloro Complexes, Phys. Chem. Chem. Phys., 8, 54285436 (2006).

[23] A.J. Anderson, R.A. Mayanovic, S. Bajt, A microbeam XAFS study of aqueous chlorozinc complexing to 430 degrees $\mathrm{C}$ in fluid inclusions from the Knaumuehle granitic pegmatite, Saxonian granulite massif, Germany, Can. Min. 36, 511-524 (1998).

[24] J.P. Simonin, R. Mills, A. Perera, P. Turq, F. Tallet, Closed capillary method for the diffusion of biological macromolecules, J. Sol. Chem. 15, 1015-30 (1986). 
[25] J.F. Dufrêche, O. Bernard, S. Durand-Vidal, P. Turq, Analytical theories of transport in concentrated electrolyte solutions from the MSA, J. Phys. Chem. B 109, 9873-9884 (2005).

[26] H.S. Harned, B.B. Owen, The Physical Chemistry of Electrolyte Solutions, American Chemical Society, Reinhold Publishing Corporation, NY, 243-245 (1963).

[27] R.A. Robinson, R.H. Stokes, Electrolyte Solutions, Butterworths, London, 288-290 (1959).

[28] J.P. Simonin, L. Blum, P. Turq, Real Ionic in the Mean Spherical Approximation. 1. Simple Salts in the Primitive Model. J. Phys. Chem. 100, 18, 7704-7709 (1996).

[29] J.P. Simonin, Study of experimental-to-McMillanMayer conversion of thermodynamic excess functions, J. Chem. Soc. Faraday Trans., 92, 35193523 (1996).

[30] J.P. Simonin, Real Ionic in the Mean Spherical Approximation. 2. Pure Strong Electrolytes up to Very High Concentrations, and Mixtures, in the Primitive Model, J. Phys. Chem. B 101, 21, 4313-4320 (1997).

[31] J. E. Huheey, E. A. Keiter, R. L. Keiter, Inorganic Chemistry: Principles of Structure and Reactivity, Prentice Hall, 1997.

[32] D.R. Turner, M. Whitfield, A.G. Dickson, The equilibrium speciation of dissolved components in freshwater and seawater at $25^{\circ} \mathrm{C}$ and $1 \mathrm{~atm}$ pressure, Geochim. Cosmochim. Acta 45, 855-881 (1981).

[33] Y. Marcus, D. Maydan, Anion Exchange of Metal Complexes. VIII. The Effect of the Secondary Cation. The Zinc-Chloride System, J. Phys. Chem. 67, 979-983 (1963).

[34] R.N. Goldberg, Evaluated Activity and Osmotic Coefficients for Aqueous Solutions: Bi-Univalent Compounds of Zinc, Cadmium, and Ethylene Bis(Trimethylammonium) Chloride and lodide, J. Phys. Chem. Ref. Data, 10, 1-55 (1981).

[35] M.F. Jones, E.A. Jones, D.F. Harmon, R.T. Semmes, A Search for Perchlorate Complexes. Raman Spectra of Perchlorate Solutions, J. Am. Chem. Soc. 83, 2038-2042 (1961). 
[36] H. Ohtaki, T. Yamaguchi, M. Maeda, X-Ray Diffraction Studies of the Structure of Hydrated Divalent Transition-Metal Ions in Aqueous Solution, Bull. Chem. Soc. Jpn. 49, 701-708 (1976).

[37] P.S. Salmon, M.C. Bellissent-Funel, G.J. Herdman, The dynamics of aqueous $\mathrm{Zn}^{2+}$ solutions: a study using incoherent quasi-elastic neutron scattering, J. Phys. Condens. Matter 2, 4297-4309 (1990).

[38] M. Maeda, T. Ito, M. Hori, G. Johansson, The Structure of Zinc Chloride Complexes in Aqueous Solution, Z. Naturforsch. 51a, 63-70 (1996).

[39] D.J. Harris, J.P. Brodholt, D.M. Sherman, Zinc Complexation in Hydrothermal Chloride Brines: Results from ab Initio Molecular Dynamics Calculations, J. Phys. Chem. A 107, 1050-1054 (2003).

[40] J. Barthel, H. Krienke, W. Kunz, Physical Chemistry of Electrolyte Solutions, Modern Aspects, Steinkopff Springer, Berlin, 1998. 
